\title{
Research on Evaluation of Ecological Carrying Capacity of Qinghai Province Based on PSR Model
}

\author{
Xiaoyong $\mathrm{Li}^{1}$, Haifa $\mathrm{Jia}^{1,2^{*}}$, and Falan $\mathrm{Zhao}^{1,2}$ \\ ${ }^{1}$ College of Civil Engineering, Qinghai University, Xining, Qinghai, 810016, China \\ ${ }^{2}$ Qinghai Provincial Key Laboratory of Energy-saving Building Materials and Engineering Safety, Xining, Qinghai, 810016, China
}

\begin{abstract}
Quantitative research and evaluation of ecological carrying capacity in Qinghai Province is in line with the requirements of the 13th five-year plan, and is of practical significance to the construction of ecological civilization in Qinghai Province. Based on the PSR model, this paper constructs the evaluation index system of ecological carrying capacity from three aspects of pressure, state , and response, uses the entropy method to determine the index weight coefficient, and comprehensively evaluates the changing trend of ecological carrying capacity since the 12th Five Year Plan of Qinghai Province from the perspectives of economy, society, resources, and environment. The results show that: from 2011 to 2019, the comprehensive evaluation of ecological carrying capacity in Qinghai Province shows a trend of first decreasing and then increasing, the response index evaluation increases greatly, the state index evaluation shows a fluctuating growth, and the pressure index is relatively stable. Based on this, the PSR model and entropy method are typical and quantifiable, which can provide a reference for Qinghai provincial government to control the ecological environment, study ecological sustainability and delimit the ecological red line.
\end{abstract}

\section{Introduction}

The early exploration of carrying capacity was mainly in the field of ecology. In 1921, Parker and Burgess put forward the concept of carrying capacity in the Journal of ecology. Carrying capacity refers to the highest limit of the possible number of individual organisms in a specific environment[1]. Later, the term carrying capacity was extended to many fields, and some related concepts appeared, such as population carrying capacity, resource carrying capacity, environmental carrying capacity, ecological carrying capacity, and so on[2]. The establishment of the theoretical model lays a foundation for the quantitative study of ecological carrying capacity. At present, the quantitative research method of ecological carrying capacity is gradually becoming mature[3].

Ecological carrying capacity is an important indicator of sustainable ecological development. At present, quantitative research methods for evaluating ecological carrying capacity have been widely used at home and abroad[4]. A large number of scholars and experts have explored the ecological carrying capacity of regions at various levels. At the national level, Zhang Wenbin[5] et al., based on the perspective of self-purification capacity and pollution level of the atmosphere, water, and soil, constructed the evaluation framework of ecological carrying capacity by using the decoupling model of ecological support and pressure, and measured and evaluated the ecological sustainability of China. At the provincial level, Wang yening[6] et al., based on the statistical data of resources and social economy, used the three-dimensional ecological footprint model to conduct dynamic research on 34 provincial administrative regions in China, refined the balance factor and yield factor of different types of land, depicted the spatial and temporal characteristics of per capita ecological footprint breadth and ecological footprint depth from 2009 to 2016, and discussed and analyzed the capital utilization pattern and its socio-economic influencing factors among provinces. At the city level, Zhu Yulin[7] et al., based on the PSR model, used data to construct a safety early warning evaluation index system for the ecological carrying capacity of the Chang-Zhu-Tan urban, and calculated the Chang-Zhu-Tan urban agglomeration from 2006 to 2015 using the grey-weighted correlation theory ecological carrying capacity safety index, and the safety alertness of ecological carrying capacity in each year was judged.

\section{Overview of the study area}

Qinghai Province is located in the northwestern inland of China. It borders Gansu to the northeast, Xinjiang to the west, Tibet to the southwest, and Sichuan to the southeast. It has abundant water resources and is known as the "Water Tower of China". Qinghai Province has many ecological function zones, the most famous ones are the Sanjiangyuan, Qilian Mountains, and Qinghai Lake ecological function zones, which provide important ecological protection for China and neighboring countries. Therefore, the ecological function areas of Qinghai

*Corresponding author's e-mail: jiahf2014@qhu.edu.cn 
Province are of great significance in China and even Southeast Asia.

\section{Research methods and data}

\subsection{PSR model}

The PSR model was first applied to Environmental Science in 1991 when OCEO used the PSR model to build an environmental assessment index system to achieve the purpose of linking the various indicators[8]. Later generations will continue to perfect this model, and the PSR model has been widely recognized and applied internationally[9]. This paper uses the PSR model to evaluate the ecological carrying capacity of Qinghai Province to link the environment, economy and society. It has the characteristics of more comprehensiveness, objectivity, validity and authenticity, and can provide a more scientific reference for the ecological construction of Qinghai Province.

\subsection{Construction of ecological carrying capacity evaluation index system and index selection}

Based on the PSR model, the index system is divided into three levels: target level, comprehensive level, and index level, which can more directly reflect the influencing factors of ecological carrying capacity in Qinghai Province. The comprehensive layer is composed of pressure, state and response of the PSR model, and the index layer is composed of 24 selected indexes. The relationship among pressure, state, and response are of great significance to the study of ecological carrying capacity in Qinghai Province. Table 1 shows the evaluation index system of ecological carrying capacity in Qinghai Province.

\subsection{Determination of indicator weights}

The entropy method is a more objective method to determine the weight of the comprehensive evaluation index. Jia Haifa[10] et al. used the entropy method to determine the index weight when measuring and evaluating the development level of ecological civilization in Qinghai Province from 2008 to 2018. This article will not repeat the calculation process of the entropy method. If you need a detailed calculation process, please contact the author to obtain it. The index weights of this article are given in Table 1.

\subsection{Data sources}

The data in this article are mainly derived from the 20112016 "Qinghai Statistical Yearbook", "China Statistical Yearbook", "Qinghai Province Environmental Status Bulletin" and other materials and documents, as well as the Qinghai Provincial People's Government. Individual missing data are calculated using the more scientific grey forecasting method.

Table1. Evaluation index system of ecological carrying capacity in Qinghai Province

\begin{tabular}{|c|c|c|c|}
\hline Comprehensive layer & Index layer & Index attribute & Weight \\
\hline \multirow{7}{*}{ Pressure } & Sulfur dioxide emissions (10000 tons) & Negative & 0.0533 \\
\hline & Total energy production (10000 tons of standard coal) & Negative & 0.0390 \\
\hline & Total energy consumption (10000 tons of standard coal) & Negative & 0.0456 \\
\hline & Number of geological disasters (time) & Negative & 0.0200 \\
\hline & Urban registered unemployment rate $(\%)$ & Negative & 0.0510 \\
\hline & Urban sewage discharge $\left(10000 \mathrm{~m}^{3}\right)$ & Negative & 0.0461 \\
\hline & Proportion of primary industry in GDP (\%) & Negative & 0.0386 \\
\hline \multirow{8}{*}{ State } & Contribution rate of secondary industry (\%) & Positive & 0.0426 \\
\hline & Built area $\left(\mathrm{km}^{2}\right)$ & Positive & 0.0379 \\
\hline & Forest coverage $(\%)$ & Positive & 0.0379 \\
\hline & Total cultivated land resources (ha) & Positive & 0.0299 \\
\hline & Total water resources (billion cubic meters) & Positive & 0.0412 \\
\hline & Total annual precipitation (mm) & Positive & 0.0449 \\
\hline & Proportion of tertiary industry in GDP (\%) & Positive & 0.0411 \\
\hline & Wetland area (10000 ha) & Positive & 0.0268 \\
\hline
\end{tabular}




\begin{tabular}{|c|l|c|c|}
\hline \multirow{5}{*}{ Response } & Number of students in higher education (10000) & Positive & 0.0447 \\
\cline { 2 - 4 } & Library collection (10000 copies) & Positive & 0.0466 \\
\cline { 2 - 4 } & Proportion of social security in fiscal expenditure (\%) & Positive & 0.0404 \\
\cline { 2 - 4 } & Proportion of education expenditure in financial expenditure (\%) & Positive & 0.0435 \\
\cline { 2 - 4 } & Proportion of energy conservation and environmental protection & Positive & 0.0478 \\
\cline { 2 - 4 } & expenditure in financial expenditure (\%) & & Positive \\
\cline { 2 - 4 } & Harmless treatment rate of municipal solid waste (\%) & Positive & 0.0307 \\
\cline { 2 - 4 } & Urban sewage treatment rate (\%) & Positive & 0.0561 \\
\cline { 2 - 4 } & Accumulated soil erosion control area (1000 ha) & Positive & 0.0388 \\
\hline
\end{tabular}

\section{Results and analysis}

\subsection{Comprehensive evaluation of ecological carrying capacity in Qinghai Province}

Based on the PSR model and using the entropy method to calculate the weights of various indicators, it can be concluded that the comprehensive score of Qinghai Province's ecological carrying capacity from 2011 to 2019 shows a trend of first decreasing and then increasing, with an average annual growth rate of $8.8 \%$. Among them, the annual growth rate of the pressure index evaluation score is $7.1 \%$, the annual growth rate of the state index evaluation score is $10.5 \%$, and the annual growth rate of the response index evaluation score is $14.5 \%$. The comprehensive evaluation index of the annual ecological carrying capacity of Qinghai Province and its comprehensive evaluation index is shown in Figure 1.

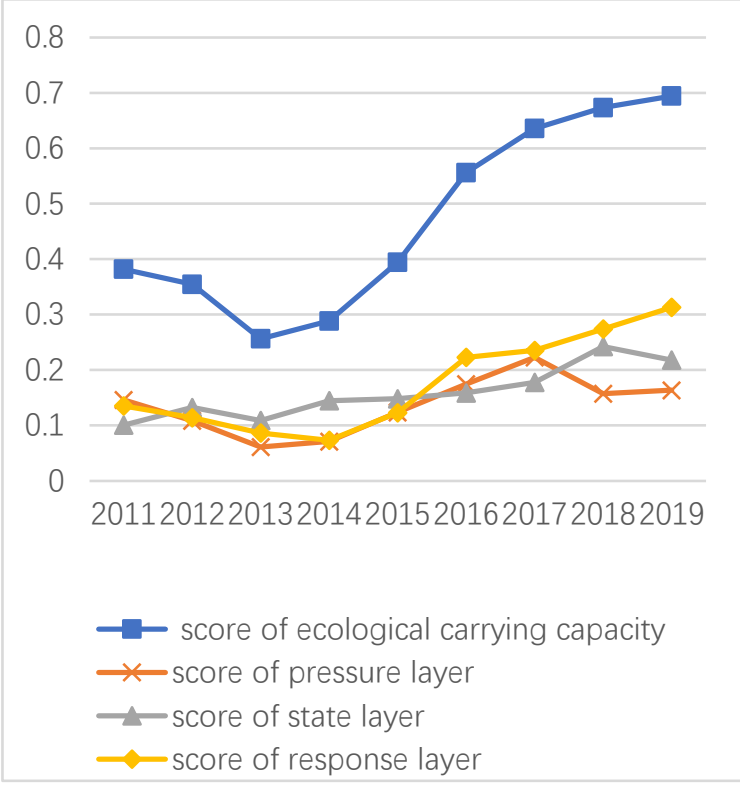

Figure 1. Change chart of comprehensive evaluation index of ecological carrying capacity and each comprehensive layer from 2011 to 2019

\subsubsection{Evaluation of pressure indicators}

From 2011 to 2019, the ecological pressure of Qinghai Province will be relieved, and the comprehensive score of ecological carrying capacity will show an overall growth trend. Among them, sulfur dioxide emissions, urban registered unemployment rate, and urban sewage emissions accounted for the largest proportion, accounting for $18.2 \%, 17.4 \%$, and $15.7 \%$ respectively. From 2011 to 2019, sulfur dioxide emissions showed a linear reduction, with a cumulative reduction of 67700 tons, with a reduced rate of $43.1 \%$ compared with 2011. In 2016, the reduction rate reached $25 \%$ compared with the previous year, with a reduction of 37100 tons. This benefited from Qinghai province's efforts to promote the elimination of small coalfired boilers and regulate the coal business market, to achieve the constraint and control of emission sources. With the acceleration of urbanization, urban population pressure, and urban sewage discharge is growing. After 2015, the discharge of urban sewage has been effectively controlled. Compared with 2015, the discharge of sewage in 2019 will be reduced by 1.48 million cubic meters.

\subsubsection{State index evaluation}

The comprehensive score of ecological bearing capacity state index in Qinghai province increased in a fluctuating way from 2011 to 2019, among which the annual total precipitation, the contribution rate of secondary industry, and the total water resources accounted for the largest proportion of the state indicators, $14.9 \%, 14.1 \%$, and $13.6 \%$ respectively. Since 2011, the total GDP of Qinghai Province has been increasing, the proportion of the secondary industry in the total GDP is still very high, accounting for more than $66 \%$ every year, and the proportion of the tertiary industry is only $0.51 \%$ every year. The good phenomenon is that the contribution rate of the secondary industry continues to decline, with an average annual decline of $3.4 \%$. It shows that the government is gradually upgrading and optimizing the industrial structure, which is beneficial to the carrying capacity of 
Qinghai Province and the development of social harmony and stability.

\subsubsection{Evaluation of response indicators}

From 2011 to 2019, a comprehensive evaluation of the ecological bearing capacity response index of Qinghai Province shows a trend of decreasing first and then increasing, with a large growth rate of $132 \%$ in 2019 compared with 2011. The proportion of the major ones is the ratio of urban sewage treatment, cumulative soil erosion control area, and energy conservation and environmental protection expenditure, accounting for $13.9 \%, 13.6 \%$, and $11.8 \%$ respectively. The obvious growth trend of response indicators shows that Qinghai provincial government attaches great importance to improving the ecological environment, actively responds to the pressure of ecological bearing capacity, puts forward various policies to protect ecological bearing capacity and implements the policies to the actual situation, and effectively improves the ecological civilization construction of Qinghai Province from the aspects of environmental economy and society.

\section{Conclusion and discussion}

Based on the PSR model, this paper selects 24 indexes from three angles of economic, environmental, and social, and uses the entropy method to determine the index weight to comprehensively evaluate the ecological carrying capacity of Qinghai Province. The results show that: from 2011 to 2019, the comprehensive evaluation of the ecological carrying capacity of Qinghai Province shows a trend of first decreasing and then increasing, and the later increasing range is large, which indicates that the ecological carrying capacity of Qinghai Province has been significantly improved. Among them, the response index evaluation performance is the most outstanding. Qinghai Province has made a good response to the current situation of ecological carrying capacity and the existing pressure. The state index evaluation shows a fluctuating rise, but the rise is small and slow, which indicates that the current situation of ecological carrying capacity in Qinghai Province is improving, but there are some obstacles; the pressure index evaluation shows a fluctuating change, specifically in the energy production and consumption and urban sewage discharge with the urbanization process is also rising, leading to the increase of ecological pressure. However, the pressure index is relatively stable, which shows that Qinghai Province has to take some measures to affect the ecological carrying capacity.

\section{Acknowledgements}

The authors gratefully acknowledge financial support from the Youth Research Fund Project of Qinghai University (Grant Nos. 2020-QSY-12).

\section{References}

1. Guo Xiurui, Mao Xianqiang, and Ran Shenghong, Research Progress of Domestic Environmental Carrying Capacity. China Population, Resources and Environment, p. 29-31(2000)

2. F Zhiming and LI Peng, The origin and development of the concept of carrying capacity: a discussion based on the perspective of resources and environment. Journal of Natural Resources, 33(9): p. 14751489(2018)

3. Liu Renzhi and Wang Chengwen, Research on Environmental Carrying Capacity Evaluation Technology. Journal of Applied Basic Science and Engineering, 17(S1): p. 92-101(2009)

4. Huang, W., et al. Study on the development and layout mode of oasis small town based on farmers' willingness survey: A case study of Zongzhai Town in China. in IOP Conference Series: Earth and Environmental Science.(2019)

5. Zhang Wenbin, Hu Jian, and Ma Yiming, Evaluation of China's ecological carrying capacity from the perspective of decoupling support and pressure. Economic Geography, 40(02): p. 181-188(2020)

6. Wang Yining, Zhou Qiang, and Wang Haowei, A dynamic study on the three-dimensional ecological footprint of 34 provincial administrative regions in China. Acta Ecologica Sinica, 40(18): p. 64346444(2020)

7. Zhu Yulin, Li Mingjie, and Gu Ronghua, Research on the safety early warning of the ecological carrying capacity of the Changzhutan urban agglomeration based on the pressure-state-response model. Resources and Environment in the Yangtze River Basin, 26(12): p. 2057-2064(2017)

8. Huang Jingnan, Ao Ningqian, and Xie Yuhang, Overview and Outlook of International Common Development Index Framework. International Urban Planning, 34(05): p. 94-101(2019)

9. Wang Guoping, et al., Construction of comprehensive disaster risk assessment index system for national parks based on PSR model. Acta Ecologica Sinica, 39(22): p. 8232-8244(2019)

10. Jia Haifa, Shao Lei, and Luo Shan, Comprehensive evaluation of ecological civilization in Qinghai Province based on entropy method and coupling coordination degree model Ecological Economy, 36(11): p. 215-220(2020) 